\title{
EDITORIAL
}

\section{1st Hypertension Research Award for authors of outstanding papers in $\mathrm{HR}$}

Hypertension Research (2011) 34, 153; doi:10.1038/hr.2010.257

$\mathrm{T}$

he Japanese Society for Hypertension (JSH) has announced the

1 winners of the 1st Hypertension Research Award. This award was newly established this year to mark significant contributions of researchers to the advancement of researches in hypertension and related studies. Every year, several awardees are selected from among the first or main authors of the articles published in Hypertension Research, an official journal of JSH, as per the recommendations of the journal's editorial committee members. For 2010, the winners are as follows:

\section{Hypertension Research Award of Excellence}

Dr Isha Shrestha

Department of Clinical Neuroscience and Therapeutics, Hiroshima University, Hiroshima, Japan (Dr Shrestha is now back in Nepal and works at Annapurna Neurological Institute)

For the contribution of 'Association between central systolic blood pressure, white matter lesions in cerebral MRI and carotid atherosclerosis', Vol. 32, No. 10, pp 869-874.

\section{Hypertension Research Award}

Dr Taku Obara

Department of Clinical Pharmacology and Therapeutics, Tohoku University Graduate School of Pharmaceutical Sciences and Medicine, Sendai, Miyagi, Japan

For the contribution of 'Uncontrolled hypertension based on morning and evening home blood pressure measurements from the J-HOME study', Vol. 32, No. 12, pp 1072-1078.

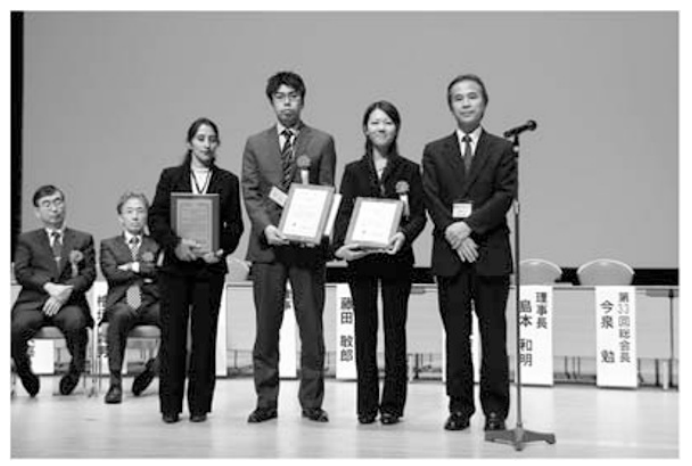

Hypertension Research Award

Dr Yumiko Watanabe

Department of Clinical Pharmacology and Therapeutics, Tohoku University Graduate School of Pharmaceutical Sciences and Medicine, Sendai, Miyagi, Japan

For the contribution of 'Accumulation of common polymorphisms is associated with development of hypertension: a 12-year follow-up from the Ohasama study', Vol. 33, No. 1, pp 129-134.

The above winners were invited to the award ceremony on 16 October in Fukuoka, Japan, held in conjunction with the JSH annual meeting, and received an award certification and supplementary prize from the then President of JSH, Prof. Kazuaki Shimamoto. 